\title{
FATORES DE RISCO ASSOCIADOS À DEISCÊNCIA APONEURÓTICA NO FECHAMENTO DA PAREDE ABDOMINAL
}

\author{
Risk factors associated to aponeurotic dehiscence in abdominal wall closure
}

\author{
Olival Cirilo Lucena da FONSECA-NETO, Rômulo VASCONCELOS, Antônio Lopes de MIRAN
}

ABCDDV/584

Fonseca-Neto OCL, Vasconcelos R, Mirian AL. Fatores de risco associados à deiscência aponeurótica no fechamento da parede abdominal. ABCD Arq Bras Cir Dig 2008;21(1):21-4

RESUMO - Racional - A deiscência aponeurótica do abdômen após intervenções cirúrgicas responde por índices de morbi-mortalidade em torno de $9 \%$ a $49 \%$ e apresenta incidência aproximada de $0,5 \%$ a 3\%. Objetivo - Identificar os fatores de risco mais prevalentes e elaborar aspecto epidemiológico do perfil dos pacientes susceptíveis à esta complicação. Métodos - Foram estudados no período de março a agosto de 200515 pacientes que estiveram internados e que apresentaram deiscência aponeurótica abdominal, provenientes da emergência ou admitidos para procedimentos eletivos. Foram pesquisados o sexo, peso e IMC, albumina sérica, linfócitos, causa do atendimento cirúrgico, co-morbidades existentes, terapias associadas, caráter da operação, incisão e fios utilizados, tempo de pós-operatório, achados cirúrgicos relacionados e total de procedimentos no sítio operatório. Resultados - Identificou-se a desnutrição e infecção do sítio cirúrgico como os principais fatores de risco, e o perfil do paciente com esta complicação foi: sexo masculino, entre 25 e 45 anos, atendido de urgência ou emergência com quadro de abdômen agudo não-traumático, desnutrido. A infecção do sítio cirúrgico, ocorreu principalmente na primeira semana de pós-operatório. Conclusão - A desnutrição e a presença de infecção cirúrgica foram os fatores de risco que mais influenciaram na ocorrência da deiscência aponeurótica.

DESCRITORES - Deiscência aponeurótica. Evisceração. Fatores de risco.

\section{INTRODUÇÃO}

Em procedimentos cirúrgicos eletivos o não fechamento do abdômen (por necessidade terapêutica ou impossibilidade) é pouco comum, o mesmo não ocorrendo em pacientes submetidos à laparotomias de urgência ou emergência. Entretanto, ao ser realizada a re-síntese, o cirurgião defronta-se com grande desafio: impedir sua reabertura ${ }^{1}$.

A deiscência aponeurótica pode ser parcial ou total, com eliminação do conteúdo abdominal, também chamada de evisceração. As conseqüências dependem diretamente da sua extensão, podendo não ocorrer nada importante, ou promover distúrbios respiratórios, perda hídrica, comprometimento da peristalse, lesões viscerais e infecções, necessidade de re-operações, entre outras ${ }^{4,6,22}$. Além dos problemas relacionados ao paciente, há aumento do tempo de internamento e custos para os sistemas de saúde ${ }^{21}$.

A incidência da ruptura da ferida operatória varia de $0,5 \%$ a $3 \%$, permanecendo inalterada desde os anos 50, apesar dos avanços científicos observados no último século ${ }^{2,4,20}$.

É papel de todo cirurgião reconhecer os fatores de risco

Trabalho realizado no Serviço de Cirurgia Geral e do Trauma do Hospital da Restauração - SUS - Recife, PE, Brasil

Endereço para correspondência: Olival Cirilo Lucena Fonseca Neto - E-mail: olivalneto@globo.com para a deiscência da aponeurose, afim de evitá-los ou minimizá-los, prevenindo assim sua ocorrência. Entretanto, a falta de significância clínica dos ensaios experimentais dificulta o estudo destes fatores de forma independente. Possivelmente eles atuam de forma sinérgica, superando a capacidade da ferida em manter sua integridade. Dentre tais fatores destacam-se: anemia, hipoalbuminemia e desnutrição, obesidade, neoplasias, icterícia, uremia, uso de corticóides, assistência ventilatória, técnica cirúrgica e material empregados, contaminação e infecção, vascularização entre outros ${ }^{8,22}$.

Até os dias atuais não existem estudos controlados e randomizados capazes de identificar a importância isolada de cada um destes fatores, verificando-se tendência da literatura em destacar os aspectos mecânicos como os principais causadores.

A observação de pacientes em pós-operatório de operações eletivas e de urgência ou emergência que apresentavam deiscência parcial ou evisceração no primeiro mês, bem como a inexistência de estudo que avaliasse a prevalência de tais fatores, motivou este trabalho que tem por objetivos identificar os fatores de risco mais prevalentes e elaborar diagnóstico epidemiológico do perfil dos pacientes susceptíveis à esta complicação. 


\section{MÉTODOS}

Trata-se de um estudo descritivo, exploratório e transversal utilizando a abordagem quantitativa. Todos os dados foram coletados após termo de consentimento livre e esclarecido apresentado a todos os pacientes ou seus responsáveis legais e terem sido assinados.

Este estudo foi desenvolvido no Serviço de Cirurgia Geral Dr. Manoel Geraldo Viana do Hospital da Restauração, Recife, PE, Brasil com pacientes vítimas de trauma e portadores de afecções cirúrgicas abdominais eletivas ou de urgência entre os meses de março a agosto de 2005 e que apresentaram deiscência aponeurótica de sutura de feridas cirúrgicas durante seu internamento. Foram excluídos pacientes com ruptura do plano músculo-aponeurótico de causa traumática. Após a seleção, a amostra resultou em 15 pacientes.

Para a análise da prevalência dos fatores de risco, coleta dos dados foi realizada a partir de formulário próprio, idealizado para a pesquisa, contendo informações sobre cada indivíduo, dados nutricionais, dados clínicos e cirúrgicos relativos à primeira laparotomia e em relação à primeira relaparotomia (Figura 1).

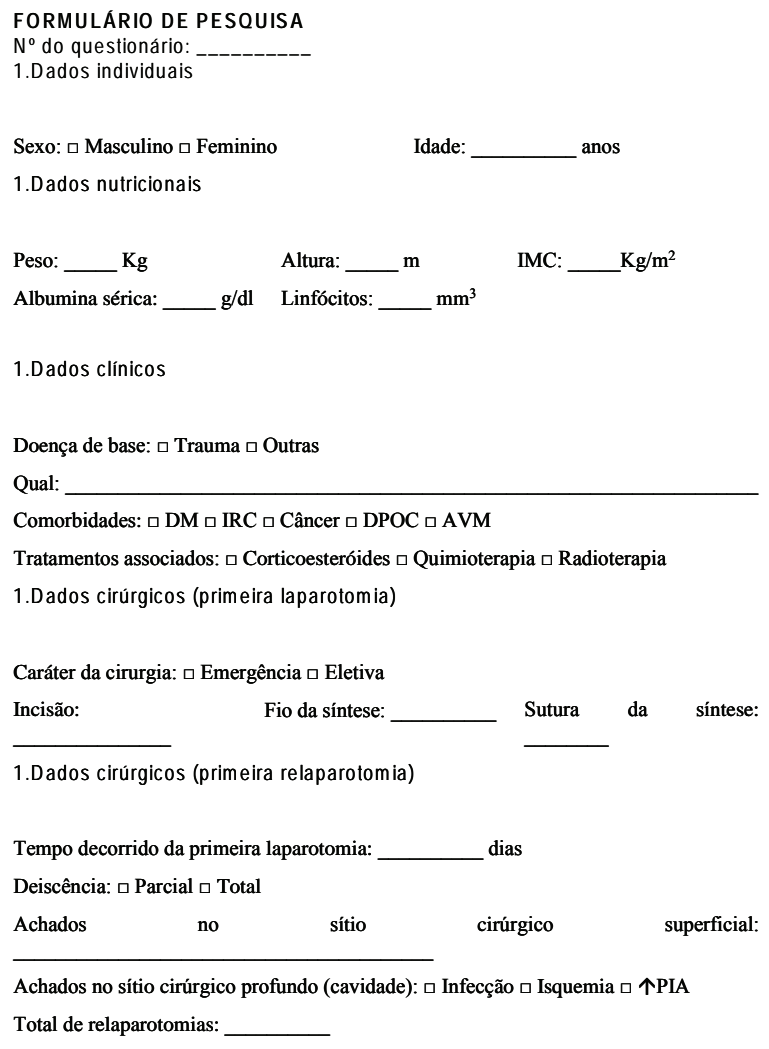

FIGURA 1 - Protocolo da pesquisa

Foram estudados o sexo, peso e IMC, albumina sérica, linfócitos, causa do atendimento cirúrgico, co-morbidades existentes, terapias associadas, caráter da operação, incisão e fios utilizados, tempo de pós-operatório, achados cirúrgicos relacionados e total de procedimentos no sítio operatório.
A partir dos dados coletados, realizou-se análise buscando associar a influência de um fator em relação ao outro.

\section{RESULTADOS}

A idade variou entre 21 e 92 anos, com predominância na faixa etária de 20 anos. Quatro indivíduos apresentaram idade superior a 60 anos.

Nenhum paciente apresentou IMC acima de 30; 13,3\% estavam abaixo de $21 ; 46,7 \%$ entre 21 e 25 e os demais (40\%) entre 26 e 30.

Em relação à dosagem de albumina sérica, todos os 15 pacientes apresentavam valores inferiores à $3,5 \mathrm{~g} / \mathrm{dL}$. Foi observado que $26,7 \%$ tinham contagem inferior a 900 células/ $\mathrm{mm}^{3}$, indicando desnutrição severa, 20\% situaram-se entre 900 e 1.200 células $/ \mathrm{mm}^{3}$, indicando desnutrição moderada e $26,7 \%$ mantiveram-se entre 1.200 e 1.500 células $/ \mathrm{mm}^{3}$, indicando desnutrição leve. Os demais (26,7\%) apresentaram contagem normal (superior a 1.500 células $/ \mathrm{mm}^{3}$ ) dando total de 73,4\% dos pacientes com algum grau de desnutrição.

Apenas 20\% dos pacientes estudados apresentavam algum distúrbio respiratório (assistência ventilatória mecânica ou doença pulmonar obstrutiva crônica), sendo $13,3 \%$ os que se encontravam em assistência ventilatória mecânica durante a evisceração e $6,7 \%$ os portadores de doença pulmonar obstrutiva crônica.

Também $20 \%$ apresentavam câncer e estavam na faixa etária de 60 anos ou mais.

Somente $2(13,4 \%)$ não tinham sido submetidos à laparotomia mediana - um a acesso paramediano e outro bicostal e os demais $(86,6 \%)$ a acesso mediano.

Em todos foi utilizado fio inabsorvível e monofilamentar para fechamento aponeurótico, tendo sido aplicada em 1 dos casos tela (também do mesmo material).

Apenas 1 dos 15 pacientes foi submetido à sutura com pontos separados $(6,7 \%)$, tendo em todos os demais sido realizada sutura contínua.

Dos 15 pacientes, $11(73 \%)$ apresentavam infecção do sítio cirúrgico profunda localizada (abcesso cavitário) ou generalizada (peritonite), sendo $8(72,7 \%)$ e $3(27,3 \%)$ respectivamente. Outros 6 pacientes $(40 \%)$ apresentavam infecção superficial (pele ou tecido celular subcutâneo), entretanto apenas 1 destes apresentaram-na na ausência de infecção cavitária (Figura 2).

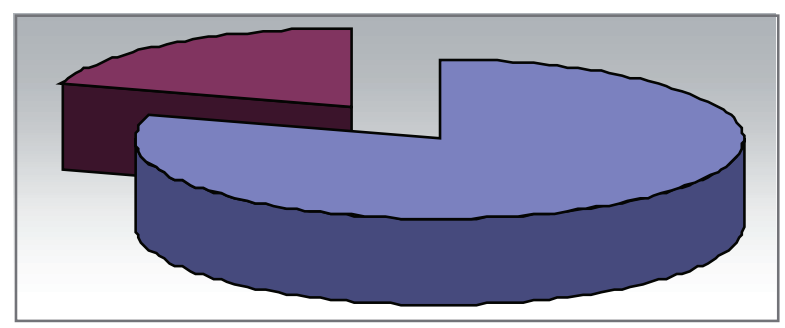

$\square$ ISC presente $\square$ ISC ausente

FIGURA 2 - Distribuição da presença de infecção do sítio cirúrgico (ISC) 
Nenhum dos pacientes que evoluíram com deiscência aponeurótica apresentava na história clínica do atendimento emergencial ou durante seu acompanhamento na enfermaria relato de choque ou isquemia da parede abdominal durante a reoperação. Portanto, o fator isquêmico não esteve presente na amostra estudada.

Dois (13,3\%) evoluíram com aumento da pressão intraabdominal. Um deles em conseqüência de distensão gasosa dos intestinos e outro do acúmulo de líquido ascítico.

Dez pacientes $(66,7 \%)$ eram do sexo masculino e 5 $(33,3 \%)$ feminino sendo que $13(86,7 \%)$ foram submetidos à operações em caráter de urgência ou emergência. A doença de base mais prevalente neste grupo foi o abdômen agudo inflamatório ou obstrutivo $(61,5 \%)$ e inflamatório $(62,5 \%)$.

O tempo entre a primeira operação e a deiscência aponeurótica foi em $8(53,3 \%)$ na primeira semana, $6(40 \%)$ na segunda e apenas 1 paciente $(6,7 \%)$ após 15 dias de pós-operatório. Em média, os pacientes apresentaram deiscência no $8^{\circ}$ dia de pós-operatório, com mínimo de quatro e máximo de 18 dias.

Quanto ao tipo de deiscência (total ou parcial), 10 (66.7\%) apresentaram deiscências parciais, enquanto os outros $5(33,3 \%)$ evoluíram com quadro de evisceração.

Apenas 4 pacientes $(26,7 \%)$ não apresentaram necessidade de nova intervenção cirúrgica para resolução da deiscência. Os outros $11(73,3 \%)$ tiveram que ser reoperados, variando de 1 a 3 novas operações (média 1,8) até a completa resolução do problema ou óbito, o que ocorreu com 3 pacientes.

\section{DISCUSSÃO}

Os cuidados com o paciente cirúrgico iniciados no pré-operatório, continuado durante o transoperatório e principalmente no pós-operatório visam minimizar os fatores capazes de influenciar ou proporcionar o surgimento de complicações. Nenhum cirurgião está isento do risco de reabertura de ferida cirúrgica e pode ser prevenida apenas se forem reconhecidas as suas causas.

Inúmeros fatores já foram identificados, atuando através de diversos mecanismos, e impedem que as fases do processo cicatricial se desenvolvam normalmente ou se associam à forças mecânicas que se sobrepujam à capacidade de manutenção da integridade da ferida operatória ${ }^{5,8,14,22}$.

A incidência da ruptura da ferida operatória abdominal tem média aproximada a $2 \%$ nas operações abdominais. Este valor sobe para $5 \%$ nos pacientes idosos ${ }^{2,9,10}$. Entretanto há autores ${ }^{3}$ que em estudos controlados em cobaias não comprovaram a idade como fator de risco para a deiscência aponeurótica.

A morbimortalidade associada à deiscência abdominal também têm-se mantido constante desde os anos 80 , entre $9 \%$ e $49 \%$ estando relacionada à doença de base ${ }^{4,20}$. Apesar do diabete melito, asma brônquica e insuficiência renal crônica serem classificadas como fatores de risco, não foram identificados pacientes com tais doenças na amostra estudada.

Admite-se que a técnica cirúrgica incorreta ou inadequada é a causa da maioria dos casos de deiscência ${ }^{5,22}$. Testes prospectivos e aleatórios da técnica de síntese não confirmaram que a sutura contínua predispõe à deiscência, embora o fio monofilamentar se associe a índice menor da ruptura da ferida do que o multifilamentar ${ }^{2,11,12}$.

Recentemente a nicotina tem sido também apontada dentre os fatores que predispõem à deiscência em cobaias $^{16}$.

A constatação de que grande parte dos pacientes eviscerados neste estudo estavam com baixos níveis de albumina sérica e linfócitos absolutos, é repetida na literatura a desnutrição como importante fator de risco para deiscência por privação orgânica de substrato químico essencial à reparação tecidual ${ }^{4,8,12}$.

Ao analisar as doenças associadas, $20 \%$ do pacientes apresentavam distúrbios ventilatórios ou câncer, fatos já também apontados como fatores predisponentes ${ }^{19,21}$.

Esta amostra não apresentou pacientes em uso de corticoterapia prolongada, radioterapia ou quimioterapia. Por tratar-se de hospital desprovido de clínica oncológica especializada, além de grande parte dos pacientes serem procedentes de emergência traumatológica, a ausência de indivíduos em tratamento radioterápico ou quimioterápico era esperada. A revisão bibliográfica do tema também não detectou estudos controlados capazes de mensurar a importância da corticoterapia no tema.

Os fatores técnico-cirúrgicos estudados no presente trabalho têm sido pesquisados, inclusive através de estudos controlados e confirmatórios. A técnica de diérese não influencia a incidência de deiscência aponeurótica. O fio recomendado é o inabsorvível na síntese que pode ser contínua ou em pontos separados ${ }^{15,17,18}$.

Ao analisarem-se os fatores locais, evidenciouse a importância da infecção local e do aumento da pressão intra-abdominal, que não foi mensurada nos pacientes da amostra, flagrando-se apenas dois casos onde ela encontrava-se evidentemente alterada para níveis anormalmente elevados devido à peritonite intensa, coleção líquida e diminuição reflexa da peristalse intestinal com acúmulo de gases ${ }^{13,17,19,21}$.

Dado importante é que o óbito dos 3 pacientes não estavam relacionados à complicação em estudo, mas às suas doenças de base (pneumonia hospitalar, choque séptico secundário à pancreatite necro-hemorrágica infectada e câncer gástrico avançado), apesar de não ser possível excluir-se deiscência como decorrentes dessas doenças.

\section{CONCLUSÃO}

A desnutrição e a presença de infecção cirúrgica foram os fatores de risco que mais influenciaram na ocorrência da deiscência aponeurótica. 
Fonseca-Neto OCL, Vasconcelos R, Mirian AL. Risk factors associated to aponeurotic dehiscence in abdominal wall closure. ABCD Arq Bras Cir Dig 2008;21(1):21-4

ABSTRACT - Background - Abdominal aponeurotic dehiscence post surgical procedures corresponds to 9\%-49\% morbid-mortality rates, presenting an incidence approximately to $0,5 \%-3 \%$. Aim - To identify the most prevalent risk factors and formulate an epidemiological archetype of patients susceptible to the respective complication. Methods - From March until August of 2005, fifteen hospitalized patients for emergency treatment or elective procedures, presenting abdominal aponeurotic dehiscence were submitted to the study. Items described for each person were sex, weight and BMI, serum albumin, lymphocytes, interventional reason, morbidity, associated therapies, surgical intervention description, incision and sutures, postoperative follow-ups, surgical findings related, and total of surgical interventions on the wound. Results - Malnutrition and surgical site infection were considered the main risk factors associated to aponeurotic dehiscence and the archetype patient reveals to be men, aging 25 to 45 years old, admitted as an emergency, presenting non-traumatic acute abdomen, and malnutrition. Surgical site infection occurred mainly during the first postoperative week. Conclusion - Malnutrition and surgical site infection were the most important risk factors related to the occurrence of aponeurotic dehiscence.

HEADINGS - Aponeurotic dehiscence. Evisceration. Risk factors.

\section{REFERÊNCIAS}

1. Almeida AD, Goffi FS. Laparotomias: técnica cirúrgica. 3.ed. São Paulo: Atheneu. 1990; 587-599.

Angood PB, Gingalewski CA, Andersen DK. Complicações cirúrgicas. In: Sabiston - Tratado de Cirurgia. Townsend CM, Beauchamp RD, Evers BM \& Mattox KL 16.ed. Rio de Janeiro: Guanabara Koogan 2002; 211-242.

3. Biondo-Simões MLP, Terranova O, Ioshii SO, Borsato KS, Weingärtner J, Nogueira G, Longhi P. Effects of aging on abdominal wall healing in rats. Acta Cirúrgica Brasileira 2005; 20:124-133.

4. Borile G, Valente DS, Pizzol MMD, Dreher R, Nunes CCA. Diagnóstico epidemiológico de evisceração em cirurgia geral. Revista do Colégio Brasileiro de Cirurgiões 2003; 30(5):388-390.

5. Colombo M, Maggioni A, Parma G, Scalambrino S, Milani R. A randomized comparison of continuous versus interrupted mass closure of midline incisions in patients with gynecologic cancer. Obstetrics \& Gynecology 1997; 89(5)1:684-689.

6. D'Angelo E, Giglio R, Lafontaine E, Bellemare F. Influence of abdomen on respiratory mechanics in supine rabbits. Respiration Physiology 1999; 287 -299 .

7. Denke M, Wilson JD. Avaliação do estado nutricional. In: Harrison - Medicina Interna. Fauci AS, Braunwald E, Isselbacher KJ, Wilson JD, Martin JB, Kasper DL, Hauser SL, Longo DL. v. 1, 14. ed. Rio de Janeiro: McGraw Hill 1998; 476-480.

8. Franco TR. Cicatrização. In: Clínica Cirúrgica: fundamentos teóricos e práticos. Vieira OM, Chaves CP, Manso JEF, Eulácio JMR. 1.ed. Rio de Janeiro: Atheneu 2000; 81-87.

9. Hodgson NCF, Malthaner RA, Ostye T. Currente practice of abdominal fascial closure: a survey of Ontario gerneral surgeons. Journal Canadien de Chirurgie 2001; 44(5):366-370.

10. Lenharo S, Mantovani M. Estudo comparativo do fechamento da incisão mediana abdominal por planos e com o uso de pontos subtotais e tela de poligalactina 910, em ratos. Acta Cirúrgica Brasileira 1998; 13(2), São Paulo.

11. Longo DL. Abordagem do paciente com câncer. In: Harrison - Medicina Interna. Fauci AS, Braunwald E, Isselbacher KJ, Wilson JD, Martin JB, Kasper DL, Hauser SL, Longo DL, v. 1, 14. ed. Rio de Janeiro: McGraw Hill 1998; $525-531$.
12. Mala FJS, Lameu EB, Vianna R. Suporte nutricional. In: Clínica Cirúrgica: Fundamentos teóricos e práticos. Vieira OM, Chaves CP, Manso JEF, Eulálio JMR. Atheneu: Rio de janeiro 2000; 59-71.

13. Monteiro MC, Ferreira RA. Tratamento ambulatorial das hérnias da parede abdominal. In: Cirurgia Ambulatorial. Souza JAG, Silva AO, v. 1, Rio de Janeiro: Atheneu 1999; 83-92.

14. Morais AAC, Paulo DNS. Afecções e condições digestivas que aumentam a incidência de complicações cirúrgicas. In: Complicações em Cirurgia do Aparelho Digestivo. Rocha PRS, Rodrigues MAG, v. 2, Rio de Janeiro: Atheneu 1998; 1-19.

15. Medeiros AC. Fios de Sutura. In: Aspectos Técnicos em Cirurgia. Margarido NF, v. 2, Rio de Janeiro: Atheneu 1999; 113-121.

16. Medeiros AC, Lima FP, Dantas Filho A M, Melo NMC, Azevedo IM. A nicotina atua como fator deletério na reparação da parede abdominal. Acta Cirúrgica Brasileira 2003; 18(1)19-23.

17. Neto JBR. Síndrome de compartimento abdominal: diagnóstico e conduta. In: Tópicos em Gastroenterologia - Obesidade e Urgências Gastroenterológicas. Rocha PRS, Coelho LGV, Diniz MTC, Nunes TA. Rio de Janeiro: Medsi 2003; 169-179.

18. Pollit DF, Beck CT, Hungler BP. Fundamentos de pesquisa: métodos, avaliação e utilização, 4. ed. Porto Alegre: Artmed, 2004.

19. Riou JA, Cohen J, Johnson H. Factors influencing wound dehiscence. American Journal of Surgery 1992; 163:324-330.

20. Rath AM, Chevrel JP. The healing of laparotomies: a bibliographic study - part two: technical aspects - Review article. Hernia 2000; 41-48.

21. Schessel ES, Ger R, AMBROSE G. The management of the post-operative disrupted abdominal wall. American Journal of Surgery 2002; 184(3):263268.

22. Soares LA, Margarido NF. Fechamento da parede abdominal. In: Aspectos Técnicos em Cirurgia. Margarido NF. v. 2, Rio de Janeiro: Atheneu, 1999; $77-86$.

Conflito de interesse: não há Fonte financiadora: não há Recebido para publicação em: 27/06/2007 Aceito para publicação em: 21/09/2007 